\title{
Effects of antioxidant treatment on seminal parameters in patients undergoing in vitro fertilization
}

\author{
Laura Gambera $^{1}$, Anita Stendardi ${ }^{1}$, Camilla Ghelardi ${ }^{1,2}$, Benedetta Fineschi ${ }^{1,2}$, Rosamaria Aini ${ }^{1}$ \\ ${ }^{1}$ A.G.I. Medica Center for Reproductive Medicine, Siena, Italy; \\ ${ }^{2}$ Department of Molecular and Developmental Medicine, University of Siena, Siena, Italy.
}

\begin{abstract}
Summary Objective: The aim of this non controlled trial was to assess whether a therapy with an antioxidant supplement may improve spermatozoa quality in terms of number, motility, morphology and a higher number of successful conceptions in patients with oligoasthenoteratozoospermia undergoing cycles of medically assisted reproduction by intracytoplasmic sperm injection (ICSI).

Materials and methods: 32 patients registered at A.G.I. Medica (Siena) medically assisted reproduction centre affected by fertility problems associated with oligoasthenoteratozoospermia were included in the study. Semen analysis were evaluated according to World Health Organization 2010, before and after treatment. Moreover, we used colorimetric tests to assess oxidative stress. After evaluating oocyte fertilisation rate and the quality of embryos obtained, data were statistically analysed.

Result: Microscopy examination after the therapy, showed a general improvement in sperm parameters (number of sperms, progressive motility, viability and normal morphology) in both baseline and capacitated; also the levels of oxidative stress was notably lower after the treatment. Morever we evaluated the outcome of the IVF treatment, the percentage of fertilization and the number of embryos obtained, all the parameters was significantly higher in the N1 group.

Conclusions: The outcomes of this trial seem to suggest that the administration of our food supplement improve semen parameters and that the evaluation of oxidative stress levels may become a diagnostic tool to assess male infertility in patients undergoing ART cycle.
\end{abstract}

KEY WORDS: Assisted reproduction technology; Embryo quality; Male infertility; Oxidative stress; Reactive oxygen species; Semen quality.

Submitted 16 May 2019; Accepted 25 July 2019

\section{INTRODUCTION}

Human infertility affects $15 \%$ of couples in their reproductive age, of which $30 \%$ is due to male factors. Alterations in spermatogenesis may be related to various clinical conditions such as varicocele, cryptorchidism, infections, nutrient deficiencies, traumas, cancer, smoke or exposure to environmental agents. However, in many cases aetiology is not clear and several studies have proved that such condition, defined as idiopathic, may be caused by oxidative stress (1).

Reactive Oxygen Species (ROS) are chemically reactive molecules that, in physiological conditions, have positive effects on sperm function (2). On the other hand, high levels of ROS may induce lipid peroxidation, damage sperm DNA (3) and protein modifications (4).

Furthermore, significant negative relations between oxidative stress (OS) parameters and semen, fertilisation rate, development of embryos and pregnancy rates have been observed (5).

Approximately $25 \%$ of men suffering from infertility show high levels of free radicals in semen (6). Increased concentration of free radicals may be due to several factors such as inflammation, cigarette smoke, ultraviolet rays, stress, alcohol, exposition to polluting agents, diets too rich in proteins and animal fats and drugs. Human ejaculate contains potential sources of ROS including leukocytes, germ cells or abnormal spermatozoa $(7,8)$. From a clinical point of view, all the aforesaid may result in lower fertilization rates, implantation failure, compromised embryo development, multiple abortions and low success in case of application of assisted reproduction techniques $(9,10)$.

Considering the high number of factors that may increase oxidative stress and thus damage spermatogenesis and nemaspermic function, antioxidant oral supplements are widely used in patients affected by infertility. In our investigation, we especially observed the therapeutic effects of a food supplement containing LArginine, Coenzyme Q10, Vitamin C and E, Inositol and active principles from plants such as Ginseng and Tribulus terrestris. These substances are known to have positive effects on the motility and mitochondrial function of spermatozoa.

Our non controlled trial was to assess whether a therapy with antioxidants may lead to actual improvement in the quality of spermatozoa as a result of a reduction in reactive oxygen species and consequently to an increase in the number of successful pregnancies in patients undergoing medically assisted reproduction.

\section{MATERIALS AND METHODS}

32 patients enrolled in this study after 12-18 months of unprotected sexual intercourses without conception. Sexual development, medical history, physical examination and serum hormone levels were normal. Serological, virological and genetic tests which are necessary to start In Vitro Fertilization (IVF) cycle were performed. All selected patients underwent microbiological analysis 
of seminal fluid to search for common bacteria Azoospermic patients and couples whose sterility was due to female factors were excluded from the study.

All the patients were informed by the doctor about the use of supplement and written informed consent was obtained from each patient.

Selected patients took a sachet a day of a food supplement (Argifast, San Marino, Italy) containing L-Arginine (3 g), Coenzyme Q10 (200 mg), Vitamin C (240 mg), Vitamin B3 (27 mg), Tribulus (Tribulus terrestris, $60 \mathrm{mg}$ ), Ginseng (Panax ginseng, $12 \mathrm{mg}$ ), Inositol (100 mg) and Vitamin E (36 mg), for a total of 65 days.

Semen samples collected by masturbation after 3-4 days of sexual abstinence were examined after liquefaction for 30 minutes at $37^{\circ} \mathrm{C}$. Volume, $\mathrm{pH}$, concentration, viability and sperm motility were assessed according to parameters of the World Health Organization (WHO) manual (11). For each sample were counted one hundred sperm and morphological characteristics of sperm organelles (nucleus, acrosomal and tail) were evaluated using an optical microscope (Zeiss, magnification 100X).

Eosin Y staining was used to detect necrotic sperm. Semen analysis was performed before (Time 0) food supplement treatment. The same tests, except for eosin test, were carried out on semen on the day of oocyte retrieval (Time 1) at the end of therapy.

Swim-up selection was performed before and after therapy to compare sperm recovery in the two steps. A sample of semen was washed in Gamete Buffer (Cook Medical ${ }^{\circledR}$, USA) and then centrifuged for 10 minutes at $161 \mathrm{rcf}$. Supernatant was removed and pellet was layered with a variable volume of medium proportional to the number and motility of the spermatozoa detected in the baseline evaluation. The sample was heated at $37^{\circ} \mathrm{C}$ for 45 minutes and then we controlled spermatozoa concentration, motility and morphology.

\section{Oxidative stress test}

Oxidative stress analysis was performed with a colorimetric test (Oxisperm ${ }^{\circledR}, A B$ Analitica, Italy) following the instructions of the manufacturer.

The Reactive Gel (RG) was liquefied in a water bath at $90^{\circ} \mathrm{C}$ for 5 minutes and then the RG temperature was reduced to $37^{\circ} \mathrm{C}$. The $R G$ was mixed with the semen sample in an Eppendorf tube in order to have a final sperm concentration of $1 \times 106 / \mathrm{mL}$ (volume proportion 1:1 semen-RG). The mix was gelified at $4^{\circ} \mathrm{C}$ for 5 minutes and then incubated for 45 minutes at $37^{\circ} \mathrm{C}$. The resulting colour of the mix was compared with the colour scheme and the corresponding OS level was estimated.

Samples were subsequently divided into two groups depending only on the OS level detected with Oxisperm ${ }^{\circledR}$ : low OS samples including levels N1 and N2 and high OS samples including levels $\mathrm{N} 3$ and $\mathrm{N} 4$.

$\mathrm{N} 1$ and N2 samples have optimal/low levels of ROS considered not able to damage cells, whereas N3 and N4 samples have levels of ROS so high that may cause pathological effects on sperms such as DNA fragmentation.

\section{Assisted reproduction procedure}

Female partners underwent ovarian stimulation through the administration of gonadotropins, which aimed to trigger the growth of multiples follicles at the same time in order to obtain a higher number of fertilizable oocytes. Oocytes were recovered from follicular fluid immediately after follicle aspiration. ICSI was performed on mature oocytes as described by Palermo et al. (12). Eighteen hours after ICSI, the presence of two pronuclei and two polar bodies was checked to verify normal fertilization. Embryos were cultured until embryo transfer, at day 3, were transferred using a catheter into the uterus in an ultrasound-guided procedure. Clinical pregnancy was confirmed by ultrasound, which showed the presence of gestational sac and foetal heart beat at the seventh week Before transfer, the embryos were evaluated morphologically and graded in order to compare embryo quality in the two different categories of patients ( $\mathrm{N} 1$ and $\mathrm{N} 2$ ). We gave them a score $(13,14)$ according to the number of cells and percentage of fragments (15).

\section{Statistical analysis}

We used Wilcoxon test (a non-parametric test) to compare the data of both groups before and after treatment. We used Mann-Whitney test (a non-parametric test) and chi-square test to compare groups with levels of OS rated $\mathrm{N} 1$ and N2. Statistical significance was set at $\mathrm{P} \leq 0.05$.

\section{RESULTS}

We selected 32 couples where the male partner was suffering from oligoasthenoteratozoospermia. Microbiological surveys did not detect the presence of common bacteria. None of the patients reported side effects and all experienced physical wellness after the 65-days therapy with antioxidants. Sperm analysis was performed before the treatment (T0) and about 65 days after therapy on the day of female oocyte retrieval (T1).

Mean sperm count, progressive motility, normal sperm morphology and eosin $\mathrm{Y}$ were below the normal range in all patients (Table 1). At Time 1, semen parameters highlighted a significant improvement in all the categories observed. Light microscopy examination after the therapy, showed a general improvement in sperm parameters (number of sperms, progressive motility, viability and normal morphology). In particular, we observed a higher quantity of spermatozoa with a well-formed nucleus and normal flagella, even though the percentage of spermatozoa with good morphology does not exceed the $25^{\text {th }}$ percentile (15). Subsequently we compared the data from the swim-up before and after therapy and it was observed a general improve of all sperm parameters examined (Table 1).

The significant improvement of parameters after swimup proved to be important in order to undergo assisted reproduction techniques since these will be the spermatozoa selected for the ICSI.

Eosin Y test on capacitated spermatozoa was carried out neither at Time 0 nor at Time 1.

A colorimetric test was performed on all the samples collected for this trial to detect the presence of free radicals in the semen before and after the treatment with antioxidants (Figure 1).

After therapy with antioxidants, it was observed that the levels of OS were notably lower and none of examined samples showed high or very high OS levels. 
Table 1.

Sperm parameters assessed in selected patients before and after treatment.

\begin{tabular}{|c|c|c|c|c|c|}
\hline & $\begin{array}{c}\text { Baseline } \\
\text { TO }\end{array}$ & $\begin{array}{c}\text { Baseline } \\
\text { T1 }\end{array}$ & $\begin{array}{c}\text { Capacitated } \\
\text { spermatozoa T0 }\end{array}$ & $\begin{array}{c}\text { Capacitated } \\
\text { spermatozoa T1 }\end{array}$ & $\begin{array}{l}\text { WHO } \\
2010\end{array}$ \\
\hline No. of spermatozoa $\left(\times 10^{6} / \mathrm{ml}\right)$ & $9.45 \pm 3.24$ & $13.39 \pm 5.06$ & $3.80 \pm 2.80$ & $5.83 \pm 4.51$ & $>15 \times 10^{6}$ \\
\hline No. of total spermatozoa $\left(\times 10^{6}\right)$ & $28.44 \pm 13.75$ & $40.61 \pm 18.80$ & $3.75 \pm 2.84$ & $5.94 \pm 4.43$ & $>39 \times 10^{6}$ \\
\hline Progressive motility (a+b\%) & $23.68 \pm 10.54$ & $37.65 \pm 10.06$ & $65.15 \pm 20.50$ & $75.87 \pm 16.76$ & $>32 \%$ \\
\hline Normal morphology (\%) & $3.43 \pm 1.68$ & $4.93 \pm 1.96$ & $11.46 \pm 5.79$ & $15.12 \pm 5.76$ & $>4 \%$ \\
\hline Eosin test (\%) & $68.43 \pm 3.83$ & $72.68 \pm 3.15$ & & & $>58 \%$ \\
\hline
\end{tabular}

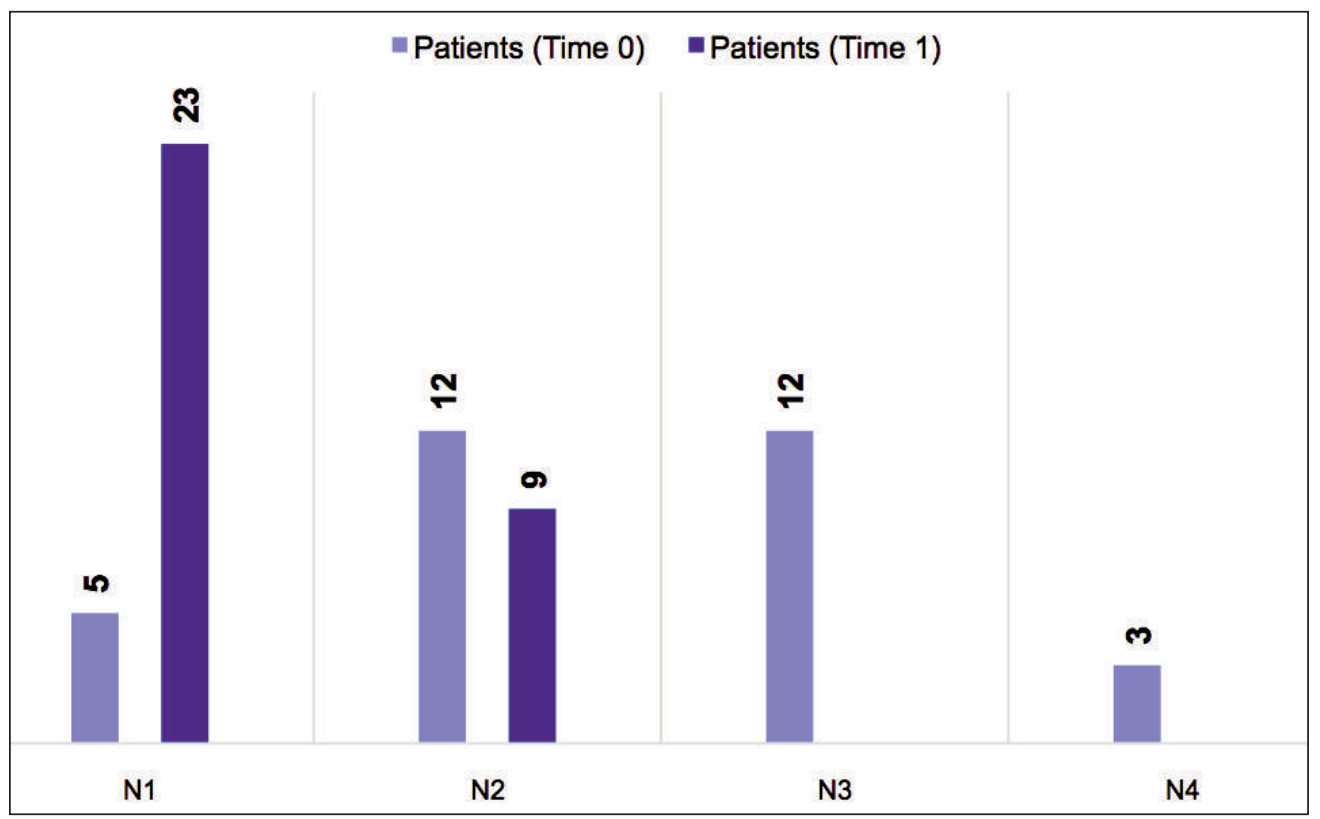

Figure 1.

Time 0: 5 patients showed no oxidative stress (N1); 12 patients had low levels of oxidative stress (N2); 12 patients had high levels of oxidative stress (N3) and 3 patients showed very high levels (N4).

Time 1: the value of 23 patients tested after the treatment was nearly zero (N1) while 9 patients proved to have N2 levels.
Then we evaluated the outcome of the IVF treatment. The percentage of fertilization and the number of embryos obtained, was significantly higher in the N1 group; our comparison highlighted a significant improvement in the embryo quality in the group showing a lower OS level. The pregnancy rate was not significative in the two groups analysed (Table 2).

\section{Conclusions}

A lower number of free radicals in semen may influence positively the development of embryos.

\section{Table 2.}

Comparison of influence of oxidative stress between group N1 and group N2 (9 patients) on the outcome of ICSI treatments.

\begin{tabular}{|lcc|}
\hline & $\mathbf{N 1}(\mathbf{n}=\mathbf{2 3})$ & $\mathbf{N 2} \mathbf{(} \mathbf{=})$ \\
\hline No. of retrieved oocytes & $5.17 \pm 2.90$ & $4 \pm 2.17$ \\
\hline \% of mature oocytes & 82.35 & 83.33 \\
\hline \% of fertilization & $92.85 *$ & $70^{*}$ \\
\hline No. of embryos obtained & $3.43 \pm 2.14 *$ & $1.77 \pm 1.30^{*}$ \\
\hline \% of cleavage & 86.81 & 76.19 \\
\hline No. of transferred embryos & $1.30 \pm 0.47$ & $1.33 \pm 0.5$ \\
\hline Embryo Score (ES) & $10.22 \pm 1.86^{*}$ & $6.94 \pm 1.8^{*}$ \\
\hline \% of pregnancies & 26.08 & 22.22 \\
\hline
\end{tabular}

Many factors may influence treatment outcome and among them reactive oxygen species (ROS) have aroused great interest in the scientific literature of the last years. ROS play a fundamental physiologic role during the various phases of reproductive process. The presence of antioxidant systems, enzymatic or not, aims to control the excessive production of reactive oxygen species and the damage they may cause (17). A lack of balance results in a condition called OS, which leads to lipid peroxidation of plasm membranes, DNA fragmentation and cell apoptosis in spermatozoa (18). The manipulation of gametes in vitro during medically assisted reproduction may be influenced by their exposure to high levels of ROS, thus suggesting the importance of using antioxidants to neutralize negative effects of the latter.

Antioxidant therapy has become quite a common treatment in case of male infertility. Number of studies describe general positive effects related to the intake of antioxidants. However, outcomes are highly variable depending on the kind of antioxidant used, its concentration or the synergy of several antioxidants administered together. Since oxidative stress seems to play a fundamental role in infertility, if antioxidant defence systems are increased, benefits comparable with those deriving from the use of traditional drugs may be obtained.

We assessed the effects of a therapy with antioxidants on semen parameters of 32 patients affected by oligoas- 
thenoteratozoospermia and oxidative stress. Morphology, motility and the number of normal spermatozoa are the parameters that significantly improved after 65 days of treatment, thus suggesting that oxidative stress may induce several alterations in the various parts of spermatozoa. The presence of ROS results in the break of plasma membranes and such effect may lead to sperm chromatin fragmentation, thus compromising male's gene pool.

The morphologic analysis of spermatozoa samples at Time 0 showed a high number of altered acrosomes, which were often missing or reacted. After the therapy with antioxidants, the nucleus was well formed with normal non-reduced acrosomes, thus suggesting that oxidative stress has negative consequences also at this level.

The assessment of semen parameters was performed also after the application of swim up method. Increased motility rates and a higher number of spermatozoa with normal morphology was observed also after such test. This improvement proved to be significant for the application of assisted reproductive techniques since these spermatozoa will be selected for ICSI.

The micro-environment of gametes and embryos during assisted reproduction is very different from physiological one: a higher oxygen concentration, temperature and $\mathrm{pH}$ surges, as well as prolonged exposition to light and media may be potential exogenous sources of oxidative stress (9). The lack of balance between antioxidant defence systems and reactive oxygen species may cause a damage that could affect the fertilization rates and embryo quality.

The impact of oxidative stress seems to have some effects also on the number of embryos obtained. Such number was double in the patients with levels of oxidative stress close to zero (N1) compared to those with low levels of OS (N2). From such data we can deduce that oxidative stress may negatively influence both fertilization process and the development of embryos. Quality assessment of embryos, which is essential during medically assisted reproduction, confirmed that the best ones develop in an environment with almost no oxidative stress. The embryo score given to evaluate embryo quality made it easy to detect the best ones. This practical, non-invasive method allowed us to select the most suitable embryo to be transferred, which led to an increase in implantation rates and to a reduction in the number of multiple pregnancies and related risks. Since the production of ROS during assisted reproduction techniques cannot be completely eliminated, suitable strategies should be adopted to minimize their effects on the treatment. The observation of abnormal levels of oxidative stress makes clinical analysis clearer, especially when reduced sperm function is not related to known illnesses. The outcomes of this trial seem to suggest that the administration of our food supplement improve semen parameters and that the evaluation of oxidative stress levels may become a diagnostic tool to assess male infertility in patients undergoing ART cycle.

\section{REFERENCES}

1. Agarwal A, Majzoub A. Role of antioxidants in assisted reproductive techniques. World J Mens Health 2017; 35:77-93.

2. Agarwal A, Nallella KP, Allamaneni SS, Said TM. Role of antioxidants in treatment of male infertility: an overview of the literature. Reprod Biomed Online. 2004; 8:616-27.
3. Aktan G, Dogru-Abbasoglu S, Küçükgergin C, et al. Mystery of idiopathic male infertility: is oxidative stress an actual risk? Fertil Steril. 2013; 99:1211-5.

4. Piomboni P, Stendardi A, Gambera L, et al. Protein modification as oxidative stress marker in normal and pathological human seminal plasma. Redox Rep. 2012; 17:227-32.

5. Tremellen K. Oxidative stress and male infertility- A clinical perspective. Hum Reprod Update. 2008; 14:243-58.

6. Zini A, San Gabriel M, Baazeem A. Antioxidants and sperm DNA damage: a clinical perspective. J Assist Reprod Genet. 2009; 26:427-32.

7. Gambera L, Campanella G, Piomboni P, et al. Ruolo di un'associazione tra antiossidanti e stimolanti immunitari naturali nel trattamento dell'astenoteratospermia con leucocitosi. Minerva Ginecol. 2007; 59:473-9.

8. Piomboni P, Gambera L, Serafini F, et al. Sperm quality improvement after natural anti-oxidant treatment of asthenoteratospermic men with leukocytospermia. Asian J Androl. 2008; 10:201-206.

9. Du Plessis SS, Makker K, Desai NR, Agarwal A. Impact of oxidative stress on IVF. Expert Rev Obstet Gynecol. 2008; 3:539-554.

10. Opuwari CS, Henkel RR. An Update on Oxidative Damage to Spermatozoa and Oocytes. Biomed Res Int. 2016; 9540142.

11. WHO laboratory manual for the Examination and processing of human semen. 2010; p.225.

12. PalermoG, Joris $H$, Devroey $P$, Van Steirteghem AC. Pregnancies after intracytoplasmatica injection of a single spermatozoon into oocyte. Lancet. 1992; 340:17-18.

13. Lee SH, Song H, Park YS, et al. Poor sperm quality affects clinical outcomes of intracytoplasmatic sperm injection in fresh and subsequent frozen-thawed cycles: potential paternal effects on pregnancy outcomes. Fertil Steril. 2009; 91:798-804.

14. Choi HW, Park YS, Lee SH, et al. Effects of maternal age on embryo quality and pregnancy outcomes using testicular sperm with intracytoplasmatic sperm injection. Clin Exp Reprod Med. 2016; 43:221-7.

15. Veek LL. An Atlas of the Human Gametes and Conceptuses. New York: Parthenon Publishing Group; 1999, pp.46-51.

16. Maizoub A, Agarwal A. Antioxidant therapy idiopathic oligoasthenoteratozoospermia. Indian J Urol. 2017; 33:207-214.

17. Aitken RJ, Clarkson JS, Fishel S. Generation of reactive oxygen species, lipid peroxidation, and human sperm function. Biol Reprod. 1989; 41:183-97.

18. Agarwal A, Majzoub A. Role of Antioxidants in Assisted Reproductive Techniques. World J Mens Health. 2017; 35:77-93.

Correspondence
Laura Gambera
lauragambera@agimedica.it
Anita Stendardi
anitastendardi@agimedica.it
Rosamaria Aini
dr.aini@agimedica.it
A.G.I. Medica Center for Reproductive Medicine
Viale Toselli 94/F, 53100 Siena, Italy
Camilla Ghelardi
c.ghelardi@agimedica.it
Benedetta Fineschi
b.fineschi@agimedica.it
Department of Molecular and Developmental Medicine, University of Siena,
Siena (Italy)

\section{Correspondence}

Laura Gambera

lauragambera@agimedica.it

Anita Stendardi

Rosamaria Ain

dr.aini@agimedica.it

A.G.I. Medica Center for Reproductive Medicine

Camilla Ghelardi

c.ghelardi@agimedica.it

Benedetta Fineschi

Department of Molecular and Developmental Medicine, University of Siena, Siena (Italy) 